\title{
The Impact of Antipsychotic Formulations on Time to Medication Discontinuation in Patients with Schizophrenia: A Dutch Registry-Based Retrospective Cohort Study
}

\author{
Arnold P. M. van der Lee ${ }^{1}(1) \cdot$ Ibrahim Önsesveren ${ }^{2} \cdot$ André I. Wierdsma $^{2} \cdot$ Roos van Westrhenen $^{3,4}$. \\ Aartjan T. F. Beekman ${ }^{1}$. Lieuwe de $\mathrm{Haan}^{5}$ - Niels C. L. Mulder²
}

Accepted: 6 March 2021 / Published online: 10 April 2021

(c) The Author(s) 2021

\begin{abstract}
Background Many patients with schizophrenia discontinue antipsychotic medication, frequently with adverse outcomes. Although different antipsychotic formulations are associated with different times to discontinuation, not much is known about discontinuation rates with oral-weekly formulations. Such a formulation of penfluridol is available in both the Netherlands and several other countries.

Objectives We aimed to investigate the impact of antipsychotic formulations on time to discontinuation, especially the oral-weekly formulation.

Methods In a large, registry-based, retrospective cohort study from 1 January 2013 to 31 December 2016, we determined the time to medication discontinuation during the follow-up period with antipsychotic formulations, including oral-daily, oral-weekly, depot, or a combination of these. Patients with schizophrenia aged between 18 and 69 years were included and stratified according to the duration of recent antipsychotic use (taking the same formulation for $\leq 60$ days or $>60$ days before follow-up: short-term or long-term recent antipsychotic use). Medication discontinuation was defined as discontinuation of current antipsychotic formulation.

Results Overall, 8257 patients were included for analyses, with $80 \%$ of patients discontinuing antipsychotic medication. Time to discontinuation was longer in those with long-term recent antipsychotic use before the follow-up period and longest for oral-daily formulations. Patterns for discontinuation of oral-weekly and depot formulations were similar, regardless of the duration of recent antipsychotic use before follow-up. More prior discontinuations were associated with shorter time to discontinuation.

Conclusions Time to discontinuation differed considerably between formulations. The duration of recent antipsychotic use was a strong predictor of time to discontinuation. While oral-daily formulations had the longest time to discontinuation in the long-term recent antipsychotic use group, discontinuation trends were similar for oral-weekly and depot formulations. An oral-weekly formulation, whose administration route is noninvasive, might therefore be considered an alternative to depot formulations.
\end{abstract}

\section{Introduction}

Schizophrenia is a serious mental disorder associated with potential deterioration in cognitive, psychosocial, and behavioral functioning [1]. There is evidence-based consensus that, in most patients, antipsychotic medication is

Arnold P. M. van der Lee and Ibrahim Önsesveren contributed equally to this work.

Arnold P. M. van der Lee

ap.vanderlee@amsterdamumc.nl

Extended author information available on the last page of the article an essential component of adequate treatment [2]. However, approximately half of patients are non-adherent [3-6]. Non-adherence is associated with several potentially severe adverse events, such as relapses, hospitalizations, and suicide attempts $[7,8]$.

Currently, long-acting injectable (LAI) antipsychotic medication is advocated in cases of non-adherence [9]; however, a substantial proportion of patients refuse it due to its invasiveness. In such cases, oral-weekly antipsychotic medication treatment with long-lasting effects (e.g. penfluridol with a weekly regimen) could be considered $[10,11]$. Penfluridol, first synthesized in 1968, is an antipsychotic 


\section{Key Points}

This is the first large, registry-based cohort study of the differences in time to medication discontinuation between different antipsychotic formulations, uniquely including an oral-weekly formulation.

Time to medication discontinuation differed substantially between antipsychotic formulations, and the duration of recent antipsychotic use was a strong predictor of it.

The discontinuation trends of the oral-weekly and depot formulations were comparable, suggesting that the oralweekly formulation could be an alternative to depot formulations in case of non-adherence and injection refusal.

drug that is deposited in fatty tissue and released slowly, resulting in long-acting properties with a half-life of $70 \mathrm{~h}$ [11]. Pharmacologically, this drug has the traditional neuroleptic properties and belongs to the diphenylbutylpiperidines [11]. Currently, penfluridol is available in the Netherlands and is being manufactured and prescribed in several other countries, including China, India, Brazil, and Israel.

Time to discontinuation is a global index for medication effectiveness, as, in a single pragmatic index, it comprises several important patient-specific aspects of treatment continuation, e.g. medication efficacy, safety, and tolerability [6, 12-15]. It can also be seen as a (proxy) measure for adherence, because of the direct relationship between adherence and treatment continuation $[6,12]$. Several observational studies have found that time to discontinuation varies between oral-daily antipsychotic medications and their LAI counterparts, showing a longer time to discontinuation using LAI $[9,12,16]$.

Although intervention and cohort studies are important in adherence research, the informed consent procedure may theoretically also introduce a bias, since only a selected group of committed patients are enrolled [17]. In an ideal scenario, such a bias is overcome in naturalistic studies involving registry data, comprising all patients in care, thus ensuring greater generalizability.

Previous studies have focused on patients, with or without prior antipsychotic medication exposure, who had started an antipsychotic medication treatment episode $[9,17]$; however, this creates a specific patient population that consists either of those who have recently been switched to a new medication or of those who are being treated for the first time. Since schizophrenia is a chronic disorder usually requiring long-term treatment, these studies may have overlooked the possible effect of the duration of recent antipsychotic use on outcomes.
Earlier studies that focused on time to discontinuation operationalized the assessment of this outcome in different ways, e.g. considering a variety of theoretical time gaps and grace periods. However, different grace periods may have significantly different impacts on discontinuation, making it difficult to compare results between studies and to draw conclusions [18].

In this study, we used Dutch health insurance company registry data to assess time to discontinuation with oraldaily, oral-weekly, and depot formulations, or a combination of these. Since health insurance is mandatory for all residents of the Netherlands, inclusion of all insured patients created a generalizable sample of patients with schizophrenia. Results were stratified on the basis of the duration of recent antipsychotic use and the outcome was operationalized using real-world medication prescription patterns.

We hypothesized that there would be considerable medication discontinuation during follow-up, with a considerable impact of the duration of recent antipsychotic use before the follow-up period and of formulation type. Finally, we hypothesized that the oral-weekly formulation, due to its noninvasive oral administration, would show noninferior discontinuation trends compared with depot formulations.

\section{Methods}

\subsection{Data Source: Dutch Computerized Health Insurance Registry Data}

All data were derived from the computerized health insurance registry data at Zilveren Kruis, a large Dutch health insurance company that at that time insured about $30 \%$ of the Netherlands' 17 million inhabitants. According to the company's internal analyses, those insured by Zilveren Kruis are representative of the Dutch population. Dutch health insurers process, check, and reimburse claims under the strict rules of the Dutch Health Insurance Authority and prevailing privacy regulations. For this study, all reimbursed healthcare claims for all clients of Zilveren Kruis provided by all the health care providers under the Dutch Health Insurance Act were available. The data consisted of all psychiatric and somatic healthcare, including prescription medication. Selection and analysis were guided by the strict rules of the Dutch privacy laws and regulations. This study was carried out according to STROBE [19].

\subsection{Study Design and Patient Selection}

For this retrospective cohort analysis, all patients who were (1) insured by the Dutch health insurer Zilveren Kruis over the whole period of 2013-2016; (2) who had been exposed to antipsychotic medication on 31 December 2015; (3) who 
had been diagnosed with schizophrenia in 2013; and (4) who were aged 18-69 years on 1 January 2013 were included.

Time to discontinuation after 31 December 2015 in the follow-up year (2016) was assessed in patients, using survival analyses (Fig. 1). Patients had been stratified according to the antipsychotic formulation they used on 31 December 2015 , combined with how long they were using that antipsychotic formulation. The amount of psychiatric treatment, as reflected by the costs of care comprising all delivered care, including (relapse-related) admissions, over 2014-2015, and patient characteristics were used to correct for the case-mix of these patients in the survival analyses.

Daily exposure to antipsychotic medication was estimated on the basis of the average prescription doses of antipsychotic medication in the dataset. Because the effective doses of antipsychotics per patients differed considerably, sensitivity analyses were performed using half and double the average prescription doses of antipsychotic medication in the dataset.

\subsection{Measures}

In the electronic supplementary material [ESM], it is explained how the average defined daily dose (DDD) representative for the Netherlands was calculated and how, for each patient, the exposure days to antipsychotic medication were determined using the Dutch DDD.

\subsection{Dependent Variable: Time to Discontinuation}

Time to discontinuation was defined as the number of days between the date on which the baseline antipsychotic formulation status had been established (31 December 2015) and the last day in 2016 before which the antipsychotic medication formulation status was switched, or on which there was no antipsychotic exposure. The 'antipsychotic status at discontinuation in 2016' means that, at discontinuation, there would be at least 1 day where there was a change to another formulation or there would be at least 1 day without antipsychotic medication. Regarding the latter, when a patient was classified as on 'no antipsychotic medication', the patient could have received antipsychotics again after this day, possibly immediately after.

\subsection{Independent Predictor Variables (Covariates)}

\subsubsection{Demographic Variables}

There were two patient characteristics: age (1 January 2013) and sex.

\subsubsection{Baseline Antipsychotic Formulation Status on $\mathbf{3 1}$ December 2015}

Antipsychotic formulation status on 31 December 2015 was determined using the Dutch DDD, and comprised the options oral-daily, oral-weekly, and depot. The antipsychotic formulation status was determined as mixed if there was an overlap between any of the possible combinations of the formulations: oral-daily, oral-weekly, or depot.

\subsubsection{Recent Antipsychotic Use}

Recent antipsychotic use was calculated by starting at baseline (31 December 2015) and counting back the number of days before that day on which a patient had been receiving the same antipsychotic formulation. Recent antipsychotic use was divided into short-term recent use ( $\leq 60$ days), and long-term recent use ( $>61$ days). We chose the 60 -day cut-off as it is consistent with definitions of newly initiated antipsychotic treatment used in other studies [6, 12].

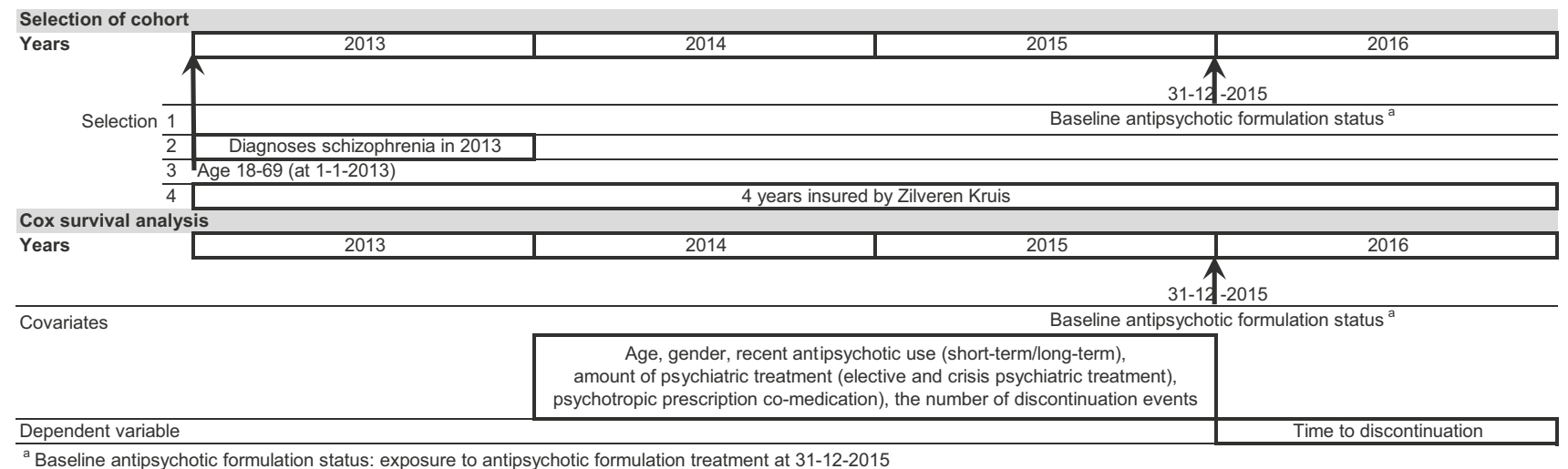

Fig. 1 Study design and patient selection 


\subsubsection{Psychiatric Treatment Over 2014-2015}

We measured the amount of elective psychiatric treatment (i.e. all outpatient non-crisis psychiatric treatment) and crisis psychiatric treatment (i.e. all crisis and inpatient psychiatric treatment) as reflected by the costs of care. The amount of psychiatric treatment was calculated using average national prices in Euros and allocated to the quarter of the starting date.

Psychotropic prescription co-medication (defined as all prescribed medication with an $\mathrm{N}$ at the first level of the Anatomical Therapeutic Chemical (ATC) code and excluding antipsychotic medication) was measured over 2014-2015 (see further explanation in the ESM). Psychotropic prescription co-medication was divided into two categories: any psychotropic prescription co-medication, or no psychotropic prescription co-medication.

The number of discontinuation events was defined as the switches in antipsychotic medication formulation status, including no antipsychotic medication exposure, and was counted over 2014-2015.

\subsection{Analysis}

The time to discontinuation was analyzed using Cox survival analyses to adjust for covariates. The Cox survival model was developed with forward selection of covariates until the fit of the model did not improve. Sensitivity analyses were performed using half and double the Dutch DDDs. All analyses were performed using SAS Enterprise guide 6.1 (SAS Institute Inc., Cary, NC, USA).

\section{Results}

\subsection{Average Defined Daily Doses (DDDs) in the Netherlands Per Formulation, and Study Population}

Table 1 presents the average DDDs used in the Netherlands. The dose of oral-daily formulations in the Netherlands was 9\% higher than the World Health Organization (WHO) DDD, and the depot dose was $45 \%$ higher. The oral-weekly dose was $25 \%$ lower than the WHO DDD. The number of patients exposed to antipsychotic medication on 31 December 2015 was 8257 .

\subsection{Patient Characteristics and Amount of Psychiatric Treatment}

Overall, the baseline antipsychotic formulation status was as follows: oral-daily formulations $(6111,74 \%)$ (Table 1), followed by depot $(1219,15 \%)$, mixed $(486,6 \%)$ and oral-weekly (441, 5\%). Sixty-four percent of patients were male, with somewhat higher percentages of males in the depot and mixed subgroups. The average age of patients was 44 years, with the oral-weekly subgroup being the oldest and the mixed subgroup being the youngest. Male patients were, on average, 5 years younger than female patients.

Before the baseline date (31 December 2015), 59\% of all patients had used the baseline antipsychotic formulation for $\leq 60$ days. The percentages of patients with recent antipsychotic use for $\leq 60$ days were $85 \%$ for the mixed formulation group, $67 \%$ for the depot subgroup, and $55 \%$ for the oral-daily and oral-weekly subgroups. Over 2014-2015, the average amount of elective psychiatric treatment (i.e. all outpatient non-crisis psychiatric treatment) was lowest in the oral-daily subgroup and highest in the oral-weekly subgroup. The average amount of crisis psychiatric treatment was also lowest in the oral-daily subgroup and highest (more than double) in the mixed subgroup.

Over the same period, the percentage of patients taking psychotropic prescription co-medication was highest in the oral-daily and mixed formulation subgroups and lowest in the depot and oral-weekly subgroups.

Over the same period, the average number of discontinuation events was highest in the oral-daily subgroup but low in the depot and mixed subgroups. The average number of discontinuation events was higher in the subgroup with shortterm recent antipsychotic use, with the highest being in the oral-daily and oral-weekly subgroups. The average number of discontinuation events was lower in the subgroup with long-term recent antipsychotic use, with the highest being in the oral-weekly and depot subgroups. In the 2016 follow-up period, only $24 \%$ had had no discontinuation (Table 2 ), with the highest percentages being in the oral-daily group (28\%), followed by the oral-weekly group (17\%), the depot group (12\%), and the mixed group (7\%). Most discontinuations in 2016 were changes to no antipsychotic medication (70\%), with the highest percentages being in the depot group (82\%), followed by the oral-weekly group (79\%), and the oral-daily group (72\%) (Table 1). The lowest percentages were in the mixed group (9\%).

\subsection{Survival Analyses}

The final Cox survival model for time to discontinuation included the following covariates: baseline antipsychotic formulation status groups, number of discontinuation events, the interaction of baseline antipsychotic formulation status groups and number of discontinuation events, age, and sex. Table 3 shows the covariates with the hazard ratios, confidence intervals, and significance levels. The other potential covariates did not improve the model.

The Cox survival curve (Fig. 2a) shows the time to discontinuation for the eight baseline antipsychotic formulation 


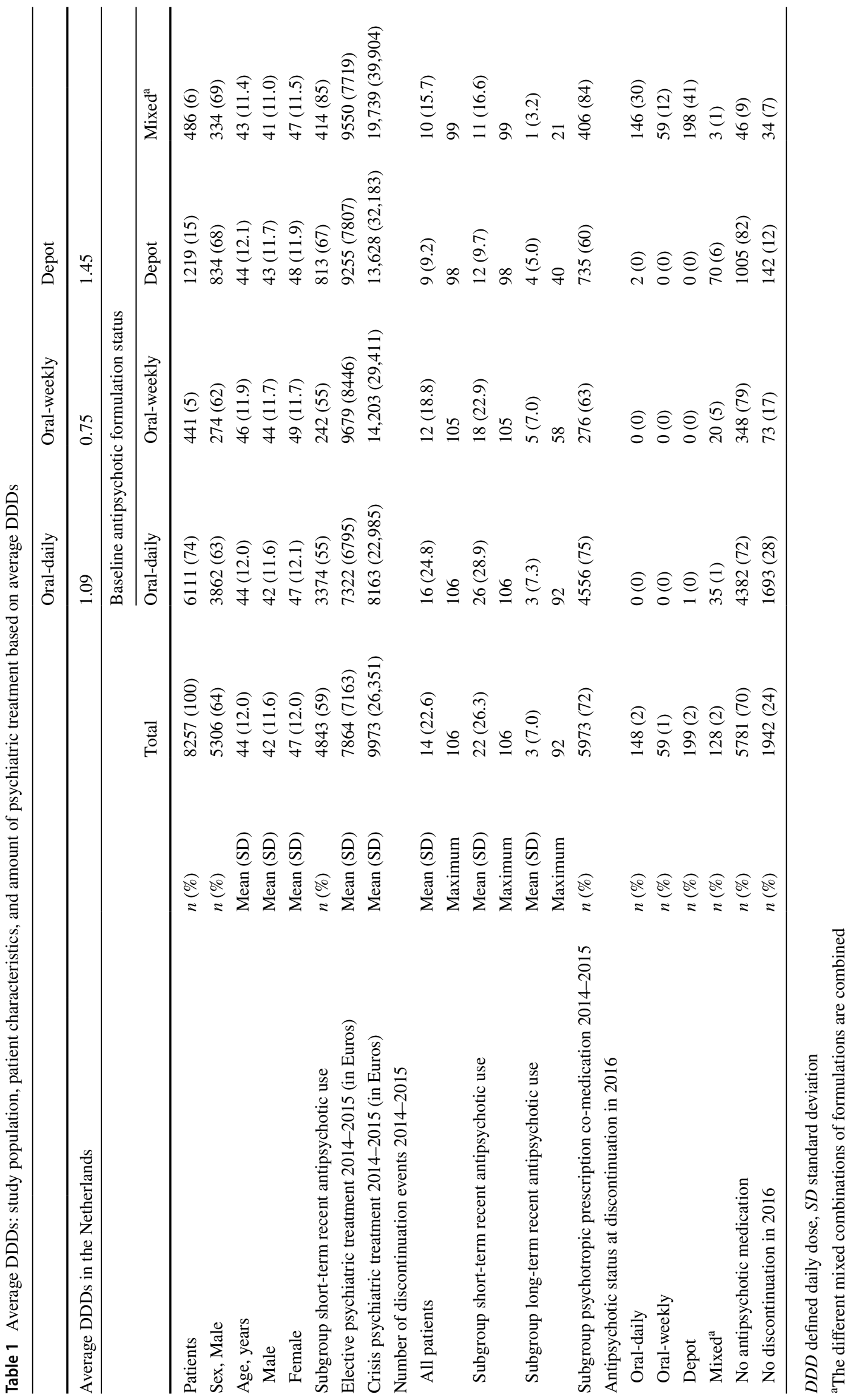


Table 2 Percentages of patients reaching the end of the 2016 follow-up year receiving the same antipsychotic formulation

\begin{tabular}{|c|c|c|c|c|c|}
\hline & Oral-daily & Oral-weekly & Depot & Mixed & Total \\
\hline \multicolumn{6}{|l|}{ Observed survival times } \\
\hline All & 28 & 17 & 12 & 7 & 24 \\
\hline Short-term recent antipsychotic use & 3 & 2 & 1 & 1 & 2 \\
\hline Long-term recent antipsychotic use & 58 & 35 & 32 & 42 & 53 \\
\hline \multicolumn{6}{|l|}{ Cox model-adjusted survival times } \\
\hline All & 23 & 15 & 10 & 4 & 20 \\
\hline Short-term recent antipsychotic use & 2 & 3 & 2 & 0 & 2 \\
\hline Long-term recent antipsychotic use & 51 & 29 & 26 & 29 & 46 \\
\hline
\end{tabular}

Data are expressed as percentages

status groups using the average DDDs for The Netherlands. The long-term recent antipsychotic use groups differed markedly from the short-term groups, with the oral-daily long-term group having the longest survival time, followed by the other long-term groups. The survival time for the short-term groups was much shorter, with the mixed shortterm group being the shortest and the remaining three groups following closely behind. After adjustment with the final Cox survival model, only $20 \%$ of all patients reached the end of follow-up without discontinuation, $2 \%$ in the short-term recent antipsychotic use subgroup, and $46 \%$ in the long-term recent antipsychotic use subgroup (Table 2).

To show the effect of the interaction between the number of discontinuation events and the baseline antipsychotic formulation status groups, the model is displayed with no discontinuation events in 2014-2015 (Fig. 2b) and also with 20 discontinuation events (Fig. 2c). The more discontinuation events there were in 2015-2016, the more the survival rates decreased. Survival was still the highest in the long-term recent antipsychotic use oral-daily subgroup, and the difference between this group and the other subgroups increased.

\subsubsection{Sensitivity Analyses: Half and Double the Average Dutch DDDs}

The results of the sensitivity analyses using half and double the average Dutch DDDs are in line with the results presented using the average Dutch DDDs in the main analysis. The results of the sensitivity analyses are presented in detail in the ESM.

The patient characteristics and psychiatric history of the three dose sets were comparable.

\section{Discussion}

This registry-based study was intended to determine the time to discontinuation with various antipsychotic formulations, including oral-daily, oral-weekly, and depot, or
Table 3 Cox survival model

\begin{tabular}{|c|c|c|c|}
\hline Covariate & HR & $95 \%$ HR CLs & $p$ value \\
\hline Oral-daily long-term & \multicolumn{3}{|l|}{ Reference } \\
\hline Oral-daily short-term & 7.99 & $7.37-8.66$ & $<0.0001$ \\
\hline Oral-weekly short-term & 6.60 & $5.57-7.81$ & $<0.0001$ \\
\hline Oral-weekly long-term & 1.71 & $1.38-2.11$ & $<0.0001$ \\
\hline Depot short-term & 6.86 & $6.12-7.70$ & $<0.0001$ \\
\hline Depot long-term & 1.88 & $1.62-2.19$ & $<0.0001$ \\
\hline Mixed short-term ${ }^{\mathrm{a}}$ & 14.64 & $12.81-16.73$ & $<0.0001$ \\
\hline Mixed long-term ${ }^{\mathrm{a}}$ & 1.65 & $1.19-2.31$ & 0.003 \\
\hline $\begin{array}{l}\text { Number of discontinuation } \\
\text { events 2014-2015 }\end{array}$ & 1.03 & $1.02-1.03$ & $<0.0001$ \\
\hline \multicolumn{4}{|c|}{ Interaction, number of discontinuation events with } \\
\hline Oral-daily short-term & 0.98 & $0.98-0.99$ & $<0.0001$ \\
\hline Oral-weekly short-term & 0.99 & $0.98-1.00$ & 0.0026 \\
\hline Oral-weekly long-term & 1.02 & $1.00-1.03$ & 0.0611 \\
\hline Depot short-term & 1.00 & $0.99-1.00$ & 0.1763 \\
\hline Depot long-term & 1.02 & $1.00-1.04$ & 0.0193 \\
\hline Mixed short-term ${ }^{a}$ & 0.99 & $0.98-0.99$ & $<0.0001$ \\
\hline Mixed long-term ${ }^{\mathrm{a}}$ & 1.02 & $0.95-1.10$ & 0.5094 \\
\hline Age & 1.00 & $1.00-1.00$ & 0.0117 \\
\hline Male & Reference & & \\
\hline Female & 1.17 & $1.11-1.23$ & $<0.0001$ \\
\hline
\end{tabular}

$H R$ hazard ratio, $C L s$ confidence limits

${ }^{a}$ In the Cox survival analyses, all combinations of formulations are combined into one group

various combinations of these in patients with schizophrenia. Our results show that substantial numbers of patients discontinued treatment. We showed that, overall, only $20 \%$ of patients reached the end of 1-year follow-up without discontinuing their medication. However, discontinuation of antipsychotic medication was strongly associated with the duration of recent antipsychotic use before the index period: $46 \%$ of patients with long-term recent antipsychotic use reached the end of follow-up without discontinuation, versus only $2 \%$ with short-term recent antipsychotic use. 


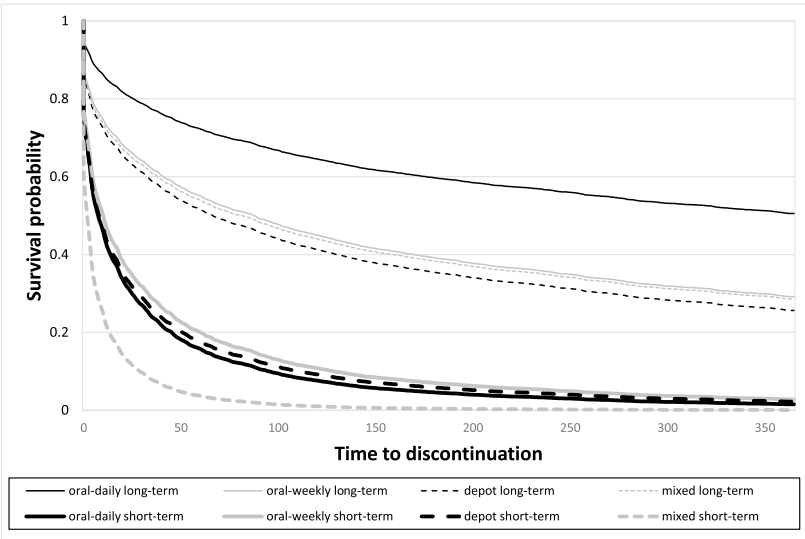

A: Cox survival model

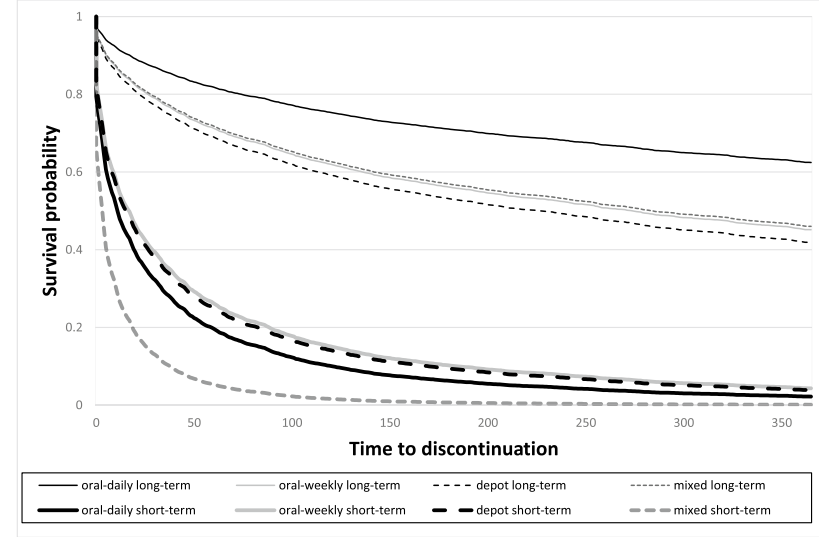

B: Using no prior discontinuation events

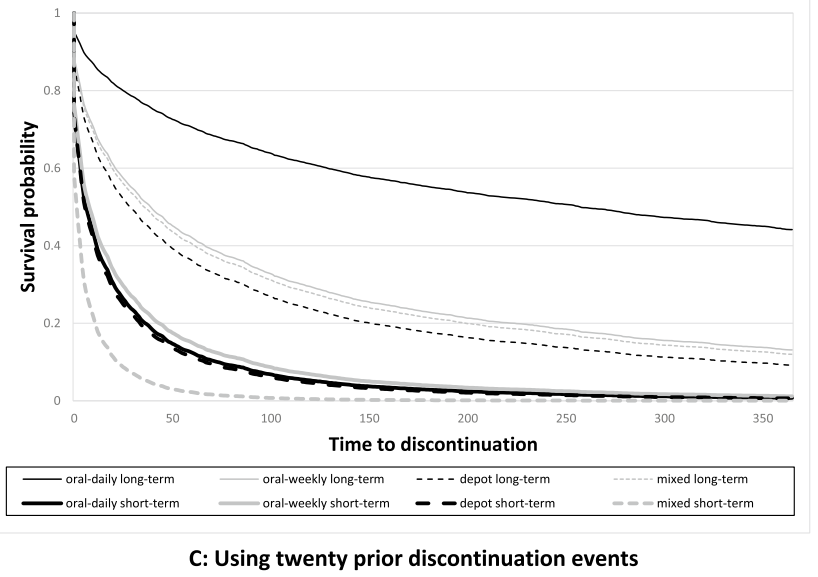

sychotic formulation (short-term recent antipsychotic use [ $\leq 60$ days] versus long-term recent antipsychotic use [ $>61$ days]). $D D D s$ defined daily doses

us, they found that time to discontinuation was longer in patients receiving depot formulations than in those patients receiving oral-daily formulations. Two factors may explain the difference in findings. First, whereas Zhu and colleagues focused on two first-generation antipsychotic medications, our study focused on formulations. To improve generalizability, our analyses included all possible antipsychotics in various formulations. However, this could have resulted in less discontinuation events, since, for instance, patients treated with oral-daily formulations could switch between different oral-daily antipsychotic medications, without discontinuing the formulation of antipsychotic medication.

Second, clinical characteristics may differ between patients initiated on different formulations across the shortand long-term recent antipsychotic use groups. Compared with patients receiving oral-daily formulations, those receiving depot formulations are more likely to have a history of non-adherence, substance abuse, poor insight, and more severe psychopathology $[16,20,21]$. As these characteristics are reflected in the number of prior discontinuations, 
our findings show that clinical characteristics have a considerable effect on time to discontinuation. With regard to long-term recent antipsychotic use, patients initiated on oral-daily formulations could have beneficial clinical characteristics, which could explain the results favoring the oraldaily formulation. To overcome potential bias by indication, we adjusted our results for potentially relevant patient and clinical characteristics (e.g. psychiatric treatment history and number of prior discontinuation events). Even so, possible residual confounding cannot be ruled out.

In this study we focused on various antipsychotic formulations, including the oral-weekly formulation. Penfluridol is the only oral-weekly antipsychotic available in this formulation. To our knowledge, penfluridol is being manufactured in several countries, e.g. China, India, Brazil, and the Netherlands. Compounding pharmacies worldwide are showing interest in this drug because of its clinical therapeutics; for example, being an oral, potent, long-acting antipsychotic drug [22]. A Cochrane review showed the safety and efficacy of penfluridol to be comparable with the depot formulation. As it was also better at 'keeping patients in treatment', penfluridol may help reduce adherence problems in patients who refuse depot formulations because of invasiveness [10, 11]. Our results show that, overall, penfluridol showed a pattern of discontinuation similar to depot formulation. These results are promising; penfluridol is prescribed mainly to a subgroup of patients who are seen as more challenging than patients receiving depot and who may refuse injections. Another interesting group in this study is the mixedformulation group. In the short-term recent antipsychotic use subgroup, mixed formulations showed the shortest time to discontinuation. There are two possible explanations for this. Either the patients using these formulations were in transition to another formulation or the treating psychiatrist decided to increase the effectiveness of therapy by starting short-term add-on therapy. On the other hand, the pattern for mixed formulations in patients with long-term recent antipsychotic use was comparable with the pattern for patients receiving depot and oral-weekly formulations, representing a more 'complex' subgroup of patients.

\subsection{Strengths and Limitations}

This study used registry data from a large health insurer whose population is representative for the Netherlands. As health insurance coverage is mandatory for Dutch citizens, financial barriers to adequate treatment are very low. Therefore, the generalizability of the results of this study is increased because the study population reflects not only the heterogeneity within the population but also includes unstable and vulnerable patients. Unlike ours, observational studies with registry data performed in other countries may, due to differences regarding healthcare policy, have focused on less vulnerable patients with sufficient financial support, potentially affecting generalizability.

Previous studies on this topic have used various methods to assess time to discontinuation, e.g. different theoretical time gaps and grace periods, due to the lack of a gold standard. These differences make it difficult to compare results. For example, different grace periods seem to have a different impact on discontinuation [18]. In our study, time to discontinuation was based on average daily maintenance doses. These average doses were determined after assessing the real prescription duration for every medication pick-up in the registry data. We believe that this provided a more valid measure of DDD in this context than the WHO DDD because of the more accurate presentation of the actual prescription duration, rather than using theoretical assumptions (Table 1). Since doses may also vary from patient to patient, we performed sensitivity analyses with half and double the calculated average DDD. These analyses showed similar patterns in the time to discontinuation. As mentioned before, studies on the associations between time to discontinuation and antipsychotic formulation in those with long-term recent antipsychotic use are limited; therefore, our findings contribute to our understanding of this important matter. To the best of our knowledge, this is also the first study in this context to focus on an oral-weekly formulation.

The following limitations have to be acknowledged. First, we used time to medication discontinuation as a measure for adherence. While this is in line with previous literature, this index focuses not only on adherence but also comprises several other important aspects [6, 12]. Previous studies have therefore used it as a global index for effectiveness rather than a measure solely of adherence [13-15]. The second limitation is that registry data contain important information on prescription pick-ups, which, however, do not necessarily equate to actual drug administration. Third, we provided no information on specific antipsychotic medications within the formulations. While it is known that differences in time to discontinuation can be associated with specific antipsychotic medications within the same formulation, our study focused not on the effect of specific antipsychotic medications on time to discontinuation but on the effect of formulation type [13]. Fourth, the oral-daily group included patients using clozapine, who represent a unique subgroup of treatmentrefractory patients. As this group comprised a small proportion of the overall sample, we do not expect it to have had a large effect on the outcomes. Fifth, even after adjustment for several relevant patient and clinical characteristics, including mental health care costs as a marker of complexity, some residual confounding (by indication) cannot be ruled out. If so, this might help explain the differences between patterns across the groups of short- and long-term recent antipsychotic use. However, as our findings demonstrate, it is important to emphasize the relevance of 'complexity' in 
the effect on time to discontinuation. For example, the group with long-term recent antipsychotic use may have been a more stable and better-treated subpopulation, which would explain the longer time to discontinuation compared with the short-term recent antipsychotic use group. This could explain why the oral-daily formulation in the long-term recent antipsychotic use group had the longest time to discontinuation. Sixth, our data are over 5 years old and do not represent the most recent medication pick-up trends in the Netherlands; however, the national treatment and prescription guidelines for schizophrenia in the Netherlands have not been changed.

Another limitation is that the oral-weekly formulation comprised only penfluridol. This may have introduced a bias since patients treated with penfluridol would reach the outcome discontinuation faster than those switched to other antipsychotics within the same formulation; patients receiving other formulations could switch between antipsychotic medications within the same formulation and still be considered as continuing their antipsychotic medication treatment. Continuation within oral-daily and depot formulations may therefore have been overestimated. Since the oral-weekly formulation and depot formulations showed a comparable pattern regarding time to discontinuation, this can be interpreted as further evidence that penfluridol is not inferior to depot formulations. It is important to note that considerably fewer patients were using penfluridol in our dataset compared with other formulations; however, we believe that there is still a sufficient number of patients using penfluridol in our analyses to adequately answer our research questions on this matter. An important issue is that penfluridol is currently not available on the market in several countries. This affects the generalizability of our results at this stage since discontinuation trends in other countries may differ due to different guidelines and policies. Finally, although naturalistic studies based on registry data have their strengths, the records in our study provided limited clinical information on patients (such as treatment orders) due to its nature. Therefore, to provide additional information about the clinical use of different formulations, we recommend further studies based on more clinical characteristics.

\section{Conclusions}

The results of the current registry-based study show that, overall, a considerable proportion of patients discontinue medication. We also found that time to discontinuation was strongly associated with the duration of recent antipsychotic use, with the longest time to discontinuation for the oraldaily formulation being in the long-term recent antipsychotic use group. Third, the patterns of time to discontinuation with the oral-weekly formulation were similar compared with the depot formulation. Therefore, the oral-weekly formulation could be considered a good alternative to depot medication in cases of non-adherence or refusal to have injections. In addition, further research on adherence should not overlook the substantial effect of the duration of recent antipsychotic use.

Supplementary Information The online version contains supplementary material available at https://doi.org/10.1007/s40263-021-00802-3.

\section{Declarations}

Funding Open access was funded by Amsterdam UMC (Vrije Universiteit Amsterdam). Zilveren Kruis Achmea supported the study in the form of salaries for Arnold van der Lee, as well as with data. Zilveren Kruis Achmea had no role in the design and conduct of the study; collection, management, analysis, and interpretation of the data; preparation, review, or approval of the manuscript; and the decision to submit the manuscript for publication.

Conflict of interest Arnold P.M. van der Lee, Ibrahim Önsesveren, André I. Wierdsma, Roos van Westrhenen, Aartjan T.F. Beekman, Lieuwe de Haan, and Niels C.L. Mulder declare that they have no conflicts of interest.

Ethics approval As the data in the study database could not be linked to individual patients, no informed consent or permission from a Medical Ethics Committee were necessary under Dutch and European laws.

Availability of data and materials The registry data that were used in this study are not publicly available due to Dutch privacy laws and regulations.

Author contributions APMvdL, IO, AIW, LdH and NCLM conceived and designed the study. APMvdL and IO collected and analyzed the data, and wrote the initial manuscript. AIW provided support during the data analysis phase. APMvdL, IO, AIW, RvW, ATFB, LdH and NCLM interpreted the results and revised the manuscript. All authors approved the final version of the manuscript and agree to be accountable for all aspects of this work.

Open Access This article is licensed under a Creative Commons Attribution-NonCommercial 4.0 International License, which permits any non-commercial use, sharing, adaptation, distribution and reproduction in any medium or format, as long as you give appropriate credit to the original author(s) and the source, provide a link to the Creative Commons licence, and indicate if changes were made. The images or other third party material in this article are included in the article's Creative Commons licence, unless indicated otherwise in a credit line to the material. If material is not included in the article's Creative Commons licence and your intended use is not permitted by statutory regulation or exceeds the permitted use, you will need to obtain permission directly from the copyright holder. To view a copy of this licence, visit http://creativecommons.org/licenses/by-nc/4.0/.

\section{References}

1. Chien WT, Yip AL. Current approaches to treatments for schizophrenia spectrum disorders, part I: an overview and medical treatments. Neuropsychiatr Dis Treat. 2013;9:1311-32. 
2. Miyamoto S, Duncan GE, Marx CE, Lieberman JA. Treatments for schizophrenia: a critical review of pharmacology and mechanisms of action of antipsychotic drugs. Mol Psychiatry. 2005;10(1):79-104.

3. Byerly MJ, Nakonezny PA, Lescouflair E. Antipsychotic medication adherence in schizophrenia. Psychiatr Clin N Am. 2007;30(3):437-52.

4. Lacro JP, Dunn LB, Dolder CR, Leckband SG, Jeste DV. Prevalence of and risk factors for medication nonadherence in patients with schizophrenia: a comprehensive review of recent literature. J Clin Psychiatry. 2002;63(10):892-909.

5. Velligan DI, Lam YW, Glahn DC, Barrett JA, Maples NJ, Ereshefsky L, et al. Defining and assessing adherence to oral antipsychotics: a review of the literature. Schizophr Bull. 2006;32(4):724-42.

6. Chan HY, Pan YJ, Chen JJ, Chen CH. Time to discontinuation of second-generation antipsychotics versus haloperidol and sulpiride in people with schizophrenia: a naturalistic, comparative study. J Clin Psychopharmacol. 2017;37(1):13-20.

7. Acosta FJ, Hernandez JL, Pereira J, Herrera J, Rodriguez CJ. Medication adherence in schizophrenia. World J Psychiatry. 2012;2(5):74-82.

8. Kane JM. Treatment adherence and long-term outcomes. CNS Spectr. 2007;12(10 Suppl 17):21-6.

9. Greene M, Yan T, Chang E, Hartry A, Touya M, Broder MS. Medication adherence and discontinuation of long-acting injectable versus oral antipsychotics in patients with schizophrenia or bipolar disorder. J Med Econ. 2018;21(2):127-34.

10. van Praag HM, Schut T, Dols L, van Schilfgaarden R. Controlled trial of penfluridol in acute psychosis. Br Med J. 1971;4(5789):710-3.

11. Soares BG, Lima MS. Penfluridol for schizophrenia. Cochrane Database Syst Rev. 2006;(2):CD002923.

12. Zhu B, Ascher-Svanum H, Shi L, Faries D, Montgomery W, Marder SR. Time to discontinuation of depot and oral first-generation antipsychotics in the usual care of schizophrenia. Psychiatr Serv. 2008;59(3):315-7.
13. Kreyenbuhl J, Slade EP, Medoff DR, Brown CH, Ehrenreich B, Afful J, et al. Time to discontinuation of first- and second-generation antipsychotic medications in the treatment of schizophrenia. Schizophr Res. 2011;131(1-3):127-32.

14. Lieberman JA, Stroup TS, McEvoy JP, Swartz MS, Rosenheck RA, Perkins DO, et al. Effectiveness of antipsychotic drugs in patients with chronic schizophrenia. N Engl J Med. 2005;353(12):1209-23.

15. Ascher-Svanum H, Zhu B, Faries D, Landbloom R, Swartz M, Swanson J. Time to discontinuation of atypical versus typical antipsychotics in the naturalistic treatment of schizophrenia. BMC Psychiatry. 2006;6:8.

16. Brnabic AJ, Kelin K, Ascher-Svanum H, Montgomery W, Kadziola Z, Karagianis J. Medication discontinuation with depot and oral antipsychotics in outpatients with schizophrenia: comparison of matched cohorts from a 12-month observational study. Int J Clin Pract. 2011;65(9):945-53.

17. Haro JM, Novick D, Belger M, Jones PB. Antipsychotic type and correlates of antipsychotic treatment discontinuation in the outpatient treatment of schizophrenia. Eur Psychiatry. 2006;21(1):41-7.

18. Decuypere F, Sermon J, Geerts P, Denee TR, De Vos C, Malfait $\mathrm{B}$, et al. Treatment continuation of four long-acting antipsychotic medications in the Netherlands and Belgium: a retrospective database study. PLoS ONE. 2017;12(6):e0179049.

19. STROBE guidelines. https://www.strobe-statement.org/.

20. Kelin K, Brnabic AJ, Newton R, Escamilla RI, Chuo LJ, Simu $\mathrm{M}$, et al. Baseline characteristics and initial treatment decisions for patients with schizophrenia at risk of treatment nonadherence. Patient Prefer Adherence. 2010;4:301-11.

21. Shi L, Ascher-Svanum H, Zhu B, Faries D, Montgomery W, Marder SR. Characteristics and use patterns of patients taking first-generation depot antipsychotics or oral antipsychotics for schizophrenia. Psychiatr Serv. 2007;58(4):482-8.

22. Zur E. Penfluridol, a unique psychiatric medicine for the treatment of chronic schizophrenia. Int J Pharm Compd. 2019;23(2):113-9.

\section{Authors and Affiliations}

\section{Arnold P. M. van der Lee ${ }^{1}$ (1) I Ibrahim Önsesveren ${ }^{2} \cdot$ André I. Wierdsma $^{2} \cdot$ Roos van Westrhenen $^{3,4}$. Aartjan T. F. Beekman ${ }^{1} \cdot$ Lieuwe de Haan $^{5}$. Niels C. L. Mulder ${ }^{2}$}

1 Department of Psychiatry, Amsterdam University Medical Centre-Location VUmc, Amsterdam, the Netherlands

2 Department of Psychiatry, Erasmus Medical Centre, Epidemiological and Social Psychiatric Research Institute, Rotterdam, the Netherlands

3 Parnassia Psychiatric Institute, Amsterdam, the Netherlands
4 Department of Psychiatry and Neuropsychology, Faculty of Health, Medicine and Life Sciences, Maastricht University, Maastricht, the Netherlands

5 Department of Psychiatry, Amsterdam University Medical Centre-Location AMC, Amsterdam, the Netherlands 